\title{
Diagnosis of Elevator Faults with LS-SVM Based on Optimization by K-CV
}

\author{
Zhou Wan, ${ }^{1}$ Shilin Yi, ${ }^{1}$ Kun Li, ${ }^{1}$ Ran Tao, ${ }^{2}$ Min Gou, ${ }^{1}$ Xinshi Li, ${ }^{2}$ and Shu Guo \\ ${ }^{1}$ Kunming University of Science and Technology, Kunming, Yunnan 650500, China \\ ${ }^{2}$ Yunnan Special Equipment Safety Inspection and Research Institute, Kunming, Yunnan, China
}

Correspondence should be addressed to Kun Li; ghfighter@163.com

Received 30 May 2015; Revised 10 November 2015; Accepted 15 November 2015

Academic Editor: William Sandham

Copyright (C) 2015 Zhou Wan et al. This is an open access article distributed under the Creative Commons Attribution License, which permits unrestricted use, distribution, and reproduction in any medium, provided the original work is properly cited.

\begin{abstract}
Several common elevator malfunctions were diagnosed with a least square support vector machine (LS-SVM). After acquiring vibration signals of various elevator functions, their energy characteristics and time domain indicators were extracted by theoretically analyzing the optimal wavelet packet, in order to construct a feature vector of malfunctions for identifying causes of the malfunctions as input of LS-SVM. Meanwhile, parameters about LS-SVM were optimized by K-fold cross validation (K$\mathrm{CV}$ ). After diagnosing deviated elevator guide rail, deviated shape of guide shoe, abnormal running of tractor, erroneous rope groove of traction sheave, deviated guide wheel, and tension of wire rope, the results suggested that the LS-SVM based on K-CV optimization was one of effective methods for diagnosing elevator malfunctions.
\end{abstract}

\section{Introduction}

With the development of modern society, elevators have achieved rapid development as tools for transporting things up and down inside high-rise buildings. While enjoying express elevator services, people have proposed more rigorous requirements for taking elevators comfortably and safely [1]. Major indicators [2] impacting if elevators can be taken with comfort include vibration of elevation compartments, noise, temperature, decoration, and starting and braking characteristics, among which the vibration is a major indicator for evaluating whether elevators can be taken comfortably [3]. Under normal circumstances, passengers will not feel uncomfortable in taking an elevator in case of relatively smaller vibration amplitude. However, passengers will have a feeling of great discomfort when the vibration reaches certain value or the frequency is up to a level to which people are sensitive [4]. To guarantee passengers' physical and mental health as well as personal safety, it is of great significance for passengers to take elevators more comfortably and safely by reducing vibration, finding out vibration source, predicting malfunctions, and recovering them promptly [5].
In case of malfunctions, vibration signals in elevators will become remarkably nonstationary with noise. Wavelet packet analysis has been widely recognized and applied in diagnosing failures of machines, especially in processing transient signals, because it is highly effective for localizing time frequency and has unique advantages in processing timevarying signals. When an elevator malfunctions, changes to the vibration signals contain abundant information about characteristics of malfunctions [6-8]. In combination with noise, elevator vibration signals were decomposed at different frequency bands by wavelet packet transform, in order to determine the energy of signals distributed in each subspace. The energy distribution was then compared with that of vibration signals in normally running system to exact information about characteristics of elevator malfunctions [9]. Besides, kurtosis of vibration signals in $Z$-axis and peak-to-peak values of vibration signals in $X / Y$-axes were taken as characteristic parameters in time domain. In this way, vibration characteristics could be extracted for malfunctions.

Support vector machine (SVM) has been extensively used and developed in the field of fault diagnosis. Tang et al. 
[10] diagnosed faults of rotating machines by LIttlewoodPaley wavelet support vector machines (LPWSVM). Shuixia et al. [11] achieved promising outcomes in classifying elevator malfunctions by diagnosing faults with kernel principal component analysis (KPCA) and SVM. Li et al. [12] put forward a model for fault diagnosis based on a genetic algorithm for hierarchically optimizing least squares support vector machines (LS-SVM), which could increase the precision of LS-SVM for predicting faults and improve self-adaptive diagnosis. Widodo and Yang [13] diagnosed faults of asynchronous motors by extracting nonlinear characteristics with SVM and achieved ideal results. In this paper, an attempt was made to optimize parameters about SVM (mainly referring to penalty parameter $c$ and kernel function $g$ ) via cross validation by integrating theories on optimal wavelet packet with SVM, so as to effectively avoid overfitting and underfitting. In other words, vibration signals were analyzed according to theories on optimal wavelet packet. By using SVM as classifier, energy characteristics and time domain indicators of vibration signals were extracted to construct fault feature vectors. They were adopted as input of SVM, and elevator malfunctions were classified with a well-trained SVM.

\section{On Safety for Taking Elevators and Mechanism}

2.1. On Safety for Taking Elevators. Elevator compartments may vibrate vertically or horizontally [1]. Major causes of their vertical vibration include vibration incurred by running a tractor, vibration of gear meshing inside a speed reducer, misalignment between axes of worm gear reducer and traction motor, and insecure fastening of tractor base with the bearing beam and nonuniform load of wire rope. All of these factors impact vertical vibration of elevator compartments. Horizontal vibration of elevator compartments is mainly caused by [14] vibration resulting from guiding system, out of tolerance perpendicularity of guide rails, out of tolerance of distance between guide rails, and out of tolerance between local gap of guide rails and steps at joints, partial distortion of guide rails, and straightness error of working surface of guide rails. These factors possibly lead compartments to horizontally vibrate in the course of up-and-down motion. Additionally, static balance of compartments is influenced by deviated suspension center and eccentric load of compartments. As a consequence, horizontal vibration is affected.

2.2. On Vibration and Mechanism of Elevator Compartments. Although there are numerous causes of compartment vibration, after analysis and investigation, the main causes [15-18] are concluded to be deviated guide rail, deviated shape of guide shoe, abnormal running of tractor, erroneous rope groove of traction sheave, deviated guide wheel and nonuniform tension of wire rope, and so on.

2.2.1. Deviated Guide Rail. Elevator compartment runs by attachment to guide rail, so horizontal vibration of an elevator is directly impacted by its guide rail. Asymmetric guide rail groove, undesirable perpendicularity of guide rail, widened or narrowed distance between guide rails and fastened bolts of guide rail brackets or clips, and so on cause horizontal vibration of compartments.
2.2.2. Deviated Shape of Guide Shoe. Attaching to guide rails, guide shoe is used to limit obliquity and horizontal displacement. It will be ineffective for reducing vibration if guide rails are adjusted to be excessively tight when an elevator is running. Consequently, the resistance for running compartments gets higher and leads to vibration. Besides, the guide shoes will become inelastic once the gap between them is regulated to be too large and thereby cause compartments to vibrate during working.

2.2.3. Abnormal Running of Tractor. High-speed shaft-driven imbalance and misaligned vibration of coupling are major causes of tractor vibration. As a tractor is rotating at a high speed, the pulse becomes an excitation source for diagnosing compartments. Errors will be caused to rope grooves and result in inaccurate dynamic balance after the tractor is worn for a long period. In addition, vibrator compartments will vibrate during running provided that speed measurement encoder is not well connected with motor or misaligned.

2.2.4. Deviated Guide Wheel. The deviation of guide wheel from the perpendicular line will be higher than $2 \mathrm{~mm}$ when the wheel is empty or fully loaded. The protection shall meet given requirements when suspended traction wheel or sprocket wheel is used. To be exact, geneva wheel shall not be seriously and nonuniformly worn to a level that its shape is changed, or else compartments will vibrate abnormally.

2.2.5. Nonuniform Tension of Wire Rope. Nonuniform tension of wire rope leads to unequal specific pressure on traction rope inside the grove of pulley wheel. Compartment will abnormally vibrate due to relative slip of rope caused by joint difference, because the rope of traction wheel is worn more quickly in case of great force [14].

\section{Signal Processing and Analysis}

In this paper, data on elevators provided by Yunnan Special Equipment Safety Inspection and Research Institute were analyzed. The data was acquired by EVA-625 elevator tester. Because the data measured about elevator running were limited, for better diagnosis, simulative samples were combined with actual samples of malfunctions to establish a sample database necessary for the training of support vector machines. Firstly, the Matlab extracts the data distribution characteristics (almost the same as authentic samples) of authentic fault samples as well as their mean value, variance, standard deviation, extremum, moment, skewness, and kurtosis, based on which it randomly generates 100 groups of simulative samples. Integrating simulative samples with authentic fault samples, it establishes the data base needed for support vector machine (SVM). 70 out of those samples are used for training and another 70 for testing.

\subsection{EVA-625 Elevator Tester}

3.1.1. EVA-625 Introduction. Aimed to record the vibrations and noises of elevators, EVA-625 elevator tester is a particularly designed high-precision recorder of acceleration 


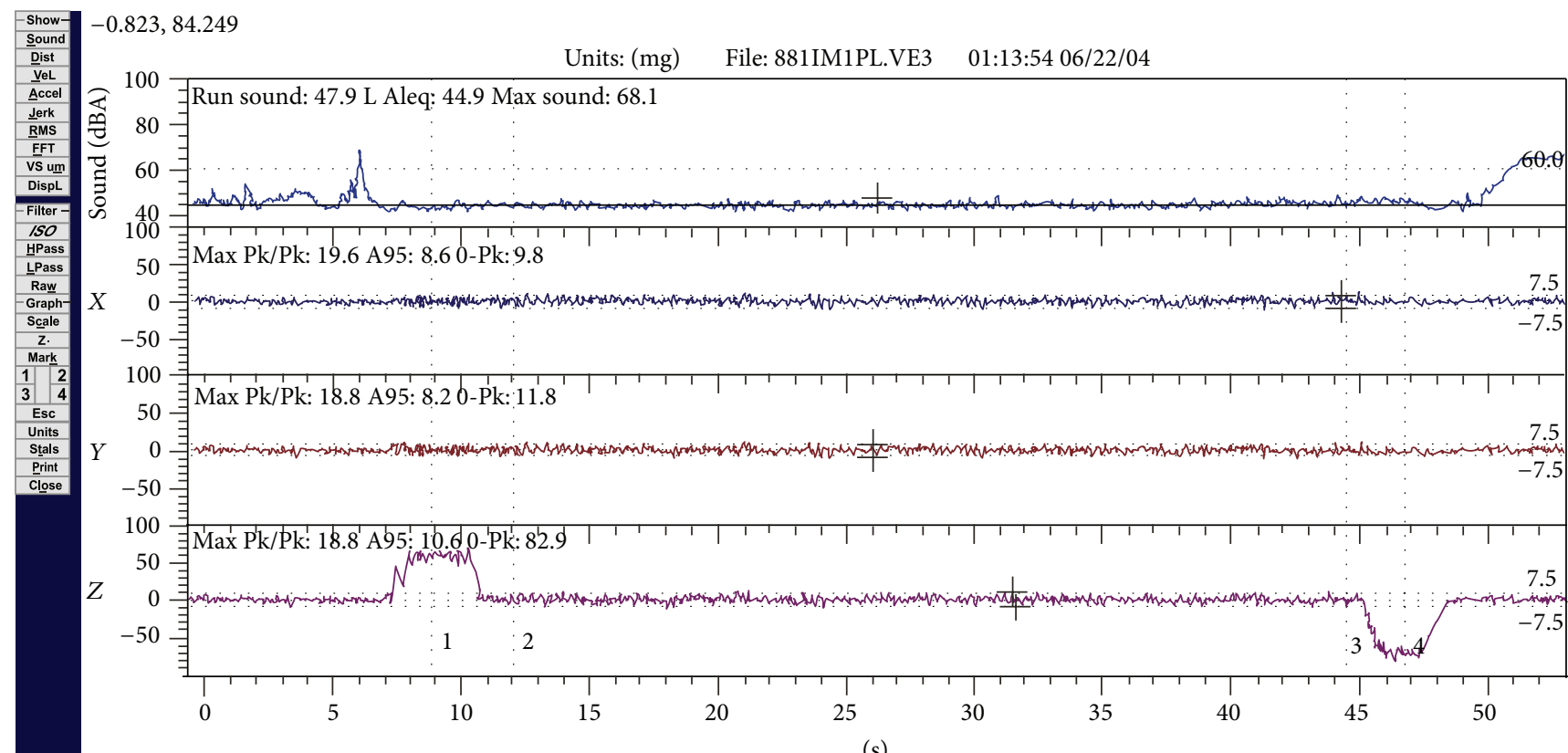

(s)

Calculate moving RMS of $X, Y, Z$ channels and display

FIGURE 1: Screenshot of variation analysis tools.
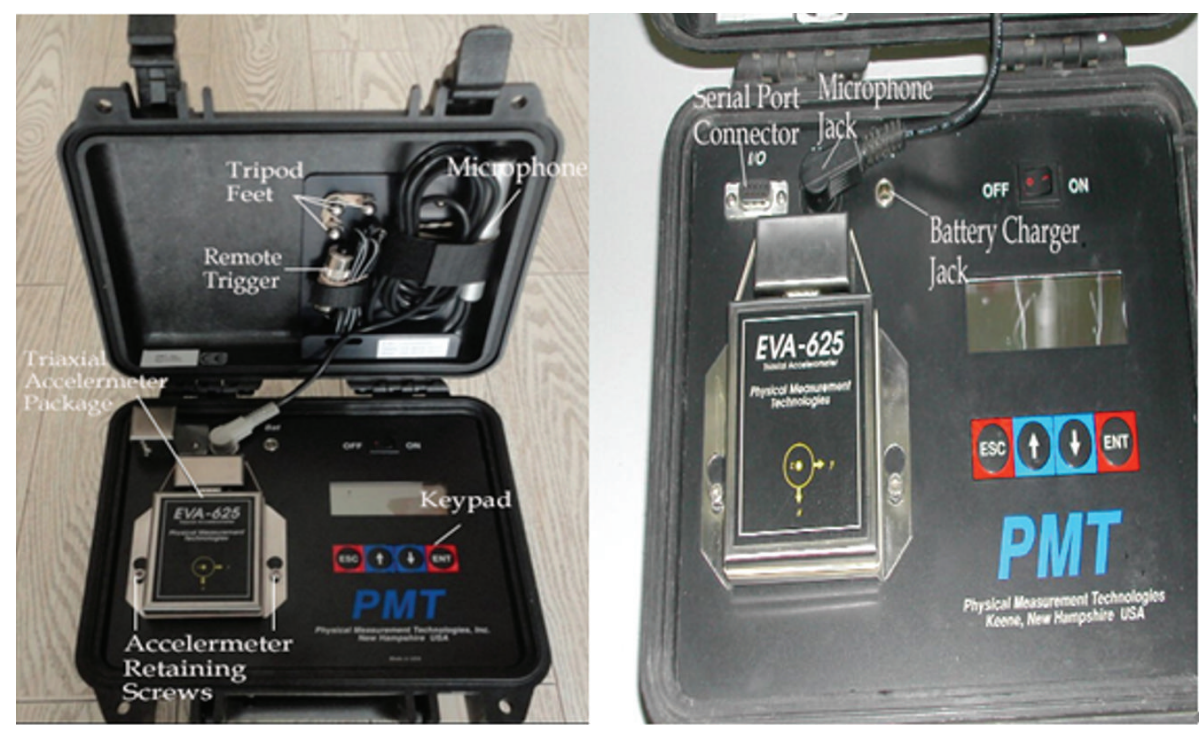

FIGURE 2: EVA-625 control and constituent parts.

and noises and the EVA system can quantize the data of acceleration as well as noises. In short, if it is put in an elevator to rise and fall, it can record the overall situation of time-variable operating state and noises of the elevator. Downloading the recorded information on the PC, you can use the apparatus-matched variation analysis tools software for analysis just as in Figure 1.

3.1.2. EVA-625 Principles and Structure. The basic structure of EVA-625 is comprised of a Triaxial Accelerometer Package, a microphone, interior circuits (digit, simulation), a LCD, a 4-key keyboard, a start/stop switch, and batteries (Figure 2):

Triaxial Accelerometer Package. It is the acceleration (vibration) sensor of EVA-625 system.

Axes of Sensitivity. The axes of sensitivity of acceleration module includes $X$-, $Y$ - and $Z$-axis. The $X$-axis tests vibration of forward and backward while the $Y$ axis measures vibration of sideways and the $Z$-axis that of vertical dimensions. 
The Microphone. It is used to collect noises while the sound track is designed at a restrictive, quickly reactive, and fidelity RMS volume to collect the volume which reaches the level of noises.

The Tachometer. The accessory allows for real time measurement and operation of escalator handrails, step speed, braking length, and its intervals.

The LCD: it is made up of 4 lines and 20 characters.

The Keyboard. It allows users to set and modify some necessary parameters while operating EVA-625 without PC.

The Start/Stop Switch. It equals the ENT and ESC button to reduce useless vibrations when beginning record model.

3.2. Wavelet Packet Analysis. Being capable of providing an accurate analytical method for signals, it divides frequency bands into multilevels, further resolving the high frequency parts that are not dispersed by multiresolution analysis and decomposing the frequency domain of the primitive into similar frequency band. In case of the sampling frequency being recognized and adequate levels being resolved, the frequency domain of each frequency band is restricted in a small scope so that similar and super-low frequencies will be in different domains. In consequences, it analyzes signals more delicately to provide more efficient approaches for extracting characteristics of signals. As an international recognized high technology to acquire and process information, wavelet packet analysis is excellently applied in many fields, such as voices, images, graphs, communications, earthquakes, biomedicines, mechanical vibrations, and computer visions.

3.3. The Energy Feature Extraction. In case of malfunctions, abundant information about features of malfunctions would be reflected from energy changes of vibration signals. Signals were decomposed by wallet packet transform at different bands, to determine the energy of signals distributed in each subspace. Then, the energy distribution was compared with the situation when the system was normally running, in order to extract information about features of elevator malfunctions. Features were exacted according to the steps as follows [9]:

(1) First of all, signals of vertical vibration acceleration were decomposed with a 4-layer $\mathrm{db} 6$-wavelet packet, and the optimal wavelet packet tree was obtained pursuant to the standard of minimum Shannon entropy, as shown in Figure 3. Next, the wavelet packets corresponding to the first two nodes on the 4 th layer, the 2 nd node on the 3 rd layer, the 2 nd node on the 2 nd layer, and the 2 nd node on the 1st layer were selected to compose the optimal wavelet packet base of signals.

(2) Signals were reconstructed with the wavelet packet bases corresponding to nodes $(4,0),(4,1),(3,1),(2,1)$, and $(1,1) . S_{40}$ was a reconstructed signal of node $(4,0)$;

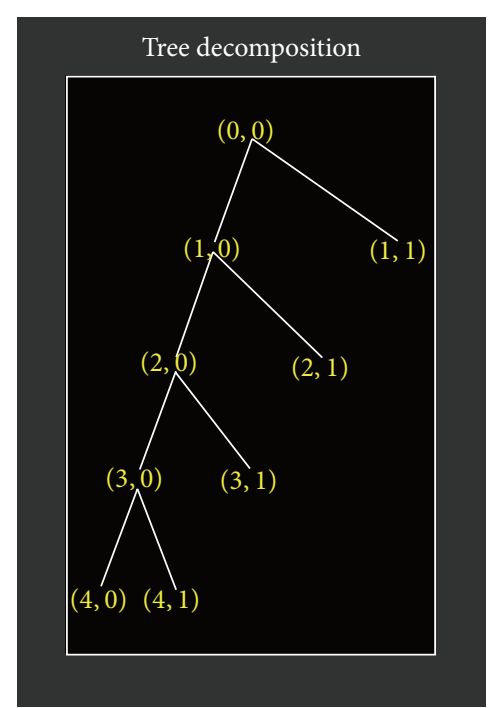

FIGURE 3: Optimal wavelet packet tree of vertical acceleration signals.

the rest could be deduced by analogy, so the overall signal reconstruction was as follows:

$$
S=S_{40}+S_{41}+S_{31}+S_{21}+S_{11}
$$

(3) The energy of each reconstructed was determined and, for $S_{40}$, the energy was calculated as follows:

$$
E_{40}=\int\left|S_{40}(t)^{2}\right| d t=\sum_{k=0}^{n}\left|X_{k}\right|^{2},
$$

where $X_{k}(k=0,1, \ldots, n)$ represents the range of $S_{40}$ at discrete points, and the energy at rest bands could be calculated in the same way.

(4) Based on abundant information about malfunctions reflected from energy of all signals at different bands, the normalized feature vector was constructed as follows:

$$
T=\left[\frac{E_{40}}{E}, \frac{E_{41}}{E}, \frac{E_{31}}{E}, \frac{E_{21}}{E}, \frac{E_{11}}{E}\right],
$$

where $E=\left(\left|E_{40}\right|^{2}+\left|E_{41}\right|^{2}+\left|E_{31}\right|^{2}+\left|E_{21}\right|^{2}+\left|E_{11}\right|^{2}\right)^{1 / 2}$.

3.4. Time Domain Analysis and Feature Extraction. The elevator vibration incurred during running may reflect basic attributes of an elevator. Changes to operating state of an elevator may cause changes to time signals of vibration and parameters described by time domain of signals. Vibration acceleration signals of a compartment were acquired from $X, Y$, and $Z$ directions in case of malfunctioning with an EVA-625 elevator tester. As remarkable changes occurred to vibration acceleration in the vertical direction when an elevator malfunctions, abnormal vibration will also take place horizontally. Therefore, kurtosis of vibration acceleration signals in the $Z$ direction and peak-to-peak values of the 
signals in $X$ and $Y$ directions were used as time domain parameters.

According to a given set of data on discreet vibration signals, the $K$ (Kurtosis) [19] was determined as follows:

$$
K=\frac{1}{N} \sum_{i=1}^{N}\left(\frac{x_{i}-\bar{x}}{\sigma_{t}}\right)^{4}
$$

where $x_{i}$ is signal value, $\bar{x}$ was mean signal value, $N$ indicated sampling length, and $\sigma_{t}$ represented standard deviation.

\section{Basic Principles of Least Squares Support Vector Machines}

Support vector machines [20-22] construct statistical learning theories according to principles of structural risk minimization when only a limited amount of samples is available. To be specific, input vectors were firstly projected from original space to high-dimension feature space through nonlinear mapping, in order to construct the optimal decision function in this high-dimension space based on principle of structural risk minimization. In this process, the calculation got less complicated as the dot product of the high-dimension feature space was replaced by kernel function of the original space. Convex quadratic programming problem was solved by the support vector machine that the extreme values obtained could be guaranteed to be the global optimal solution. In this way, the deficiency that neural networks easily became the smallest within local areas was overcome.

Having extended the standard SVM, the least squares SVM is proposed by J. A. K. SuyKens and J. Vandewalle, acquiring outstanding achievements in pattern recognition and nonlinear function fitting. The differences between LSSVM and standard SVM lie in that the loss function of an optimized object is represented by 2-norm error, inequality constraints of SVM are replaced by equality constraints, and convergence rate is increased.

Assuming that the training set is $\left\{\left(x_{1}, y_{1}\right), \ldots,\left(x_{n}, y_{n}\right)\right\}$, where $n$ is total number of samples, $x_{i} \in R^{n}$ is the $i$ th sample input, and $y_{i} \in\{-1,1\}$ is desired output of the $i$ th sample, the function of linear regression was calculated as follows:

$$
y(x)=\omega^{T} X+b,
$$

where $X=\left(x_{1}, x_{2}, \ldots, x_{n}\right)$ is sample input, $\omega=\left(\omega_{1}, \omega_{2}, \ldots\right.$, $\omega_{n}$ ) is a weighted coefficient of LS-SVM, and $b$ is a threshold.

According to criteria of structural risk minimization (SRM) [23-25], optimized problem could be converted into

$$
\min \frac{1}{2}\|\omega\|^{2}=\frac{1}{2} c \sum_{i=1}^{n} \xi_{i}^{2}
$$

The constraint is as follows:

$$
y_{i}=\langle\omega \cdot X\rangle+b+\xi_{i},
$$

where $c$ is a tolerable penalty coefficient $(c>0)$ for controlling the degree of penalties on samples beyond the calculation error, $\xi_{i}$ is a relaxation factor, and $\langle\cdot\rangle$ is a mapping function of kernel space.
By introducing a Lagrange function, the model of regression function was obtained for LS-SVM according to KKT optimality conditions as follows:

$$
f(x)=\sum_{i=1}^{n} \alpha_{i} K\left(X, X_{i}\right)+b,
$$

where $\alpha_{i}$ is a Lagrange multiplier and $K\left(X, X_{i}\right)$ is a kernel function for calculating the inner product of sample data. In using a regression model with LS-SVM, it is necessary to properly set a coefficient of tolerable penalty and select a suitable kernel function.

4.1. Classification and Analysis of LS-SVM. In this paper, feature vectors of energy distribution of elevator vibration acceleration signals, including $E_{40}, E_{41}, E_{31}, E_{21}$, and $E_{11}$, were selected. Besides, time domains of vibration acceleration signals of a compartment in $X, Y$, and $Z$ directions were used, involving gradient in the $Z$ direction and peak-to-peak values in $X$ and $Y$ directions. Furthermore, extreme values of noise measured by a noise sensor was considered, so malfunction symptoms composed by these nine characteristic values were adopted as parameters input into SVM, in order to classify training samples. There are 70 groups training samples. At last, tested samples were classified and identified with a well-trained classifier. Malfunctions were defined as follows: normal state $=1$, deviation of elevator guide rail $=2$, deviation of shape of guide show $=3$, error of abnormal running of tractor $=4$, error of rope groove of traction sheave $=5$, deviation of guide wheel $=6$, and nonuniformity of tension of wire rope $=7$.

4.2. Parameter Optimization for LS-SVM. In the process of realizing LS-SVM, two parameters should be determined, including kernel function $g$ and penalty factor $c$. Parameters were optimized with $\mathrm{K}-\mathrm{CV}$, while the processes for searching and optimizing optimal parameters were shown in Figures 4 and 5 as follows. Firstly, parameters were sketchily searched within $2^{-10} \sim 2^{10}$ with coarse mesh, as shown in Figure 4. Then, optimal parameters $c$ and $g$ were found to range within $2^{-2} \sim 2^{10}$ and $2^{-10} \sim 2^{0}$. Subsequently, parameters were precisely searched within the sphere to find out the optimal parameter with K-CV method (Figure 5).

The searching results suggested that LS-SVM could realize the optimal precision for identifying faults when $c$ is 0.5 and $g$ equals 1.

Figure 1 offers part of the recognition results of 70 groups test samples about normal state of elevators and 6 kinds of fault types and the recognition efficiency is $92.7 \%$. It may be clearly seen from the table that the normal state of elevators, deviated elevator guide rail, deviated shape of guide shoe, abnormal running of tractor, erroneous rope groove of traction sheave, deviated guide wheel, and tension of wire rope were successfully identified with a LS-SVM classifier optimized through cross validation (Table 1). 
TABLE 1: Results for identifying elevator faults with a LS-SVM optimized by cross validation.

\begin{tabular}{|c|c|c|c|c|c|c|c|c|c|c|}
\hline \multirow{3}{*}{$\begin{array}{l}\text { Faults of tested } \\
\text { samples }\end{array}$} & \multicolumn{9}{|c|}{ Input of SVM } & \multirow{3}{*}{ Output } \\
\hline & \multicolumn{5}{|c|}{ Energy characteristics } & \multicolumn{3}{|c|}{ Time Domain characteristics } & \multirow[b]{2}{*}{$\begin{array}{l}\text { Noise extremes } \\
(\mathrm{dB})\end{array}$} & \\
\hline & $E_{40}$ & $E_{41}$ & $E_{31}$ & $E_{21}$ & $E_{11}$ & $k$ & $\begin{array}{c}\text { Peak-to-peak } \\
\text { values in } X \\
\text { direction }\left(\mathrm{m} / \mathrm{s}^{2}\right)\end{array}$ & $\begin{array}{c}\text { Peak-to-peak } \\
\text { values in } Y \\
\text { direction }\left(\mathrm{m} / \mathrm{s}^{2}\right)\end{array}$ & & \\
\hline Normal state (1) & 0.089 & 0.167 & 0.124 & 0.118 & 0.068 & 0.889546 & 0.009 & 0.01 & 47.9 & 1 \\
\hline Normal state (2) & 0.093 & 0.173 & 0.136 & 0.121 & 0.079 & 0.796675 & 0.009 & 0.012 & 47.1 & 1 \\
\hline $\begin{array}{l}\text { Deviation of } \\
\text { guide rail (1) }\end{array}$ & 0.044 & 0.242 & 0.276 & 0.171 & 0.026 & 4.139485 & 0.191 & 0.024 & 58.1 & 2 \\
\hline $\begin{array}{l}\text { Deviation of } \\
\text { guide rail (2) }\end{array}$ & 0.041 & 0.241 & 0.285 & 0.166 & 0.024 & 3.247673 & 0.178 & 0.025 & 59.5 & 2 \\
\hline $\begin{array}{l}\text { Deviation of } \\
\text { shape of guide } \\
\text { shoe (1) }\end{array}$ & 0.56 & 0.294 & 0.028 & 0.031 & 0.012 & 3.173433 & 0.021 & 0.02 & 55.6 & 3 \\
\hline $\begin{array}{l}\text { Deviation of } \\
\text { shape of guide } \\
\text { shoe (2) }\end{array}$ & 0.534 & 0.295 & 0.027 & 0.034 & 0.018 & 4.272636 & 0.025 & 0.019 & 55.2 & 3 \\
\hline $\begin{array}{l}\text { Abnormal } \\
\text { running of tractor } \\
\text { (1) }\end{array}$ & 0.545 & 0.294 & 0.028 & 0.038 & 0.022 & 2.380825 & 0.021 & 0.02 & 56.4 & 4 \\
\hline $\begin{array}{l}\text { Abnormal } \\
\text { running of tractor } \\
(2)\end{array}$ & 0.582 & 0.273 & 0.026 & 0.036 & 0.02 & 3.567978 & 0.025 & 0.018 & 55.9 & 4 \\
\hline $\begin{array}{l}\text { Error of rope } \\
\text { groove of traction } \\
\text { sheave }(1)\end{array}$ & 0.814 & 0.031 & 0.022 & 0.022 & 0.024 & 3.677453 & 0.024 & 0.022 & 57.1 & 5 \\
\hline $\begin{array}{l}\text { Error of rope } \\
\text { groove of traction } \\
\text { sheave }(2)\end{array}$ & 0.79 & 0.026 & 0.021 & 0.024 & 0.022 & 2.824331 & 0.023 & 0.022 & 56.4 & 5 \\
\hline $\begin{array}{l}\text { Deviation of } \\
\text { guide wheel (1) }\end{array}$ & 0.02 & 0.212 & 0.11 & 0.066 & 0.105 & 3.187661 & 0.124 & 0.1 & 53.8 & 6 \\
\hline $\begin{array}{l}\text { Deviation of } \\
\text { guide wheel (2) }\end{array}$ & 0.022 & 0.207 & 0.108 & 0.056 & 0.105 & 2.095107 & 0.115 & 0.1 & 54.2 & 6 \\
\hline $\begin{array}{l}\text { Uniformity of } \\
\text { tension on wire } \\
\text { rope (1) }\end{array}$ & 0.12 & 0.525 & 0.028 & 0.118 & 0.022 & 2.109227 & 0.02 & 0.019 & 55.6 & 7 \\
\hline $\begin{array}{l}\text { Uniformity of } \\
\text { tension on wire } \\
\text { rope (2) }\end{array}$ & 0.124 & 0.566 & 0.028 & 0.109 & 0.021 & 3.099955 & 0.023 & 0.02 & 55.9 & 7 \\
\hline
\end{tabular}
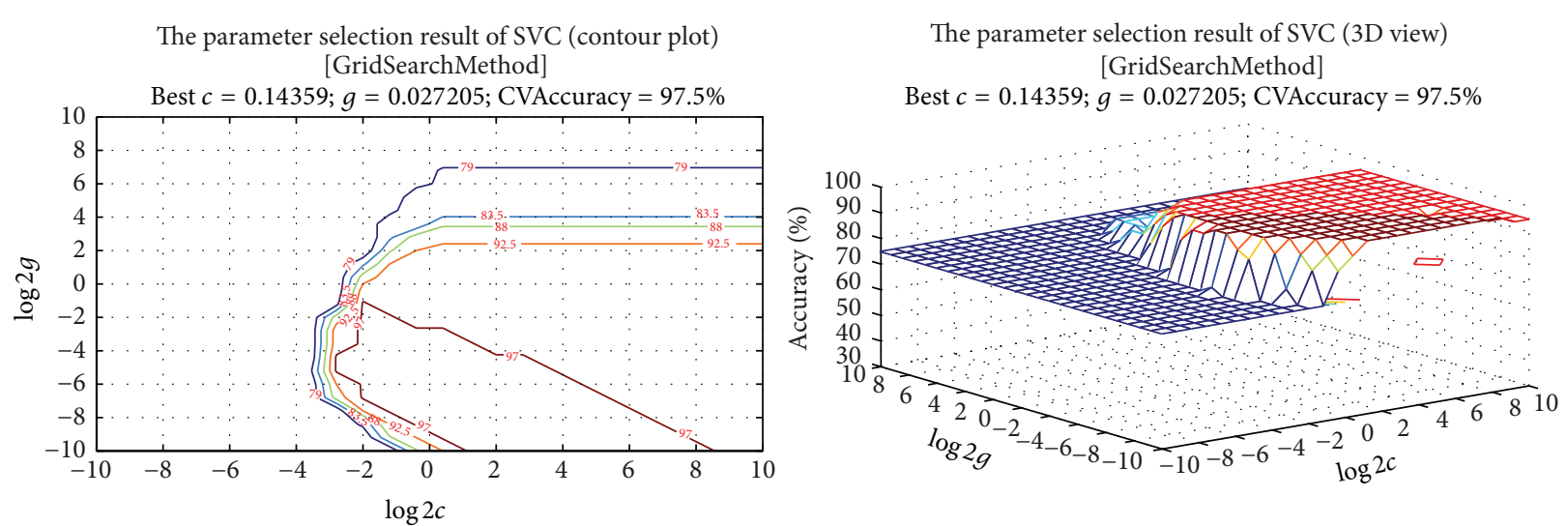

FIGURE 4: Roughly selected parameters. 

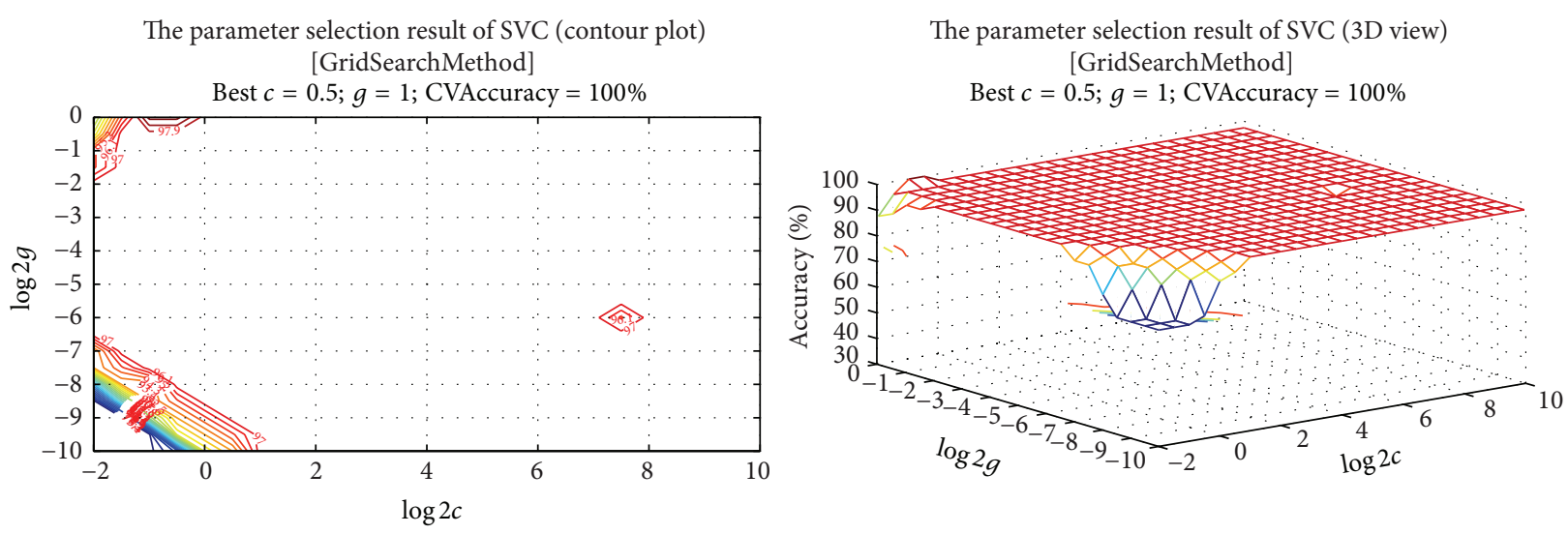

FIgURE 5: Precisely selected parameters.

\section{Conclusions}

(1) The basic idea of wavelet packet analysis is to centralize information and energy to find out laws from details and provide a more precise method for signal analysis. Thus, elevator faults were diagnosed according to theory on optimal wavelet packet with the least squares support vector machine.

(2) Feature vectors of energy distributed by elevator vibration signals and feature vectors of faults constructed by time-domain parameters were used as input of LS-SVM. Elevator malfunctions were classified and identified with a well-trained LS-SVM classifier. Parameters of LS-SVM were optimized via cross validation, in order that the classifier could classify LS-SVM most precisely.

(3) Experimental results suggested that this method was effective for identifying faults of elevators. As an advanced way for intelligently diagnosing faults, it has good prospects for application in condition monitoring and fault diagnosis.

\section{Conflict of Interests}

The authors declare that there is no conflict of interests regarding the publication of this paper.

\section{Acknowledgments}

This work is supported by the science and technology plan project of national bureau of quality inspection (2013QK104) and the science and technology plan project of quality and technical supervision bureau in Yunnan province (2013ynzjkj02).

\section{References}

[1] L. Li, A Study on the Measurement System and Technology of Fault Diagnosis for Elevators, Tianjin University, Tianjin, China, 2002.
[2] S. Jie, "The research on the influence of the elevator's comfortable sensation," China Elevator, vol. 1, pp. 43-44, 1991.

[3] Z. Changming, "The evaluation method on the comfortable sensation of elevator vibration," Construction Mechanization, vol. 6, pp. 29-32, 1988.

[4] Y. Fashan, Study on Elevator Intelligent Diagnosis System Based Multi-sensor Information Fusion Technology, Xinjiang University, Xinjiang, China, 2013.

[5] Z. Tongbo, "The research on the problem of elevator vibration," Guide of Sci-Tech Magazine, vol. 26, pp. 269-279, 2010.

[6] T. Baoguo, Y. Yunlong, and Z. Jiping, "Aeroengine fault diagnosis based on wavelet transform and neural network," Journal of Naval Aeronautical and Astronautical Uniersity, vol. 24, no. 3, pp. 241-244, 2009.

[7] T. Zan, M. Wang, G. Li, and R.-Y. Fei, "Application of wavelet packet and fuzzy ART neural network to vibration exception monitoring for grinding process," Journal of Beijing University of Technology, vol. 34, no. 7, pp. 678-707, 2008.

[8] Y. Yu and D. Wan, "Simulation research on an approach of fault detection using wavelet neural networks," Computer Simulation, vol. 17, no. 1, pp. 24-27, 2000.

[9] Y. Li and H.-H. Zhang, "Intelligent elevator detecting system based on neural network," Journal of Beijing University of Technology, vol. 36, no. 4, pp. 440-444, 2010.

[10] B.-P. Tang, F. Li, and R.-X. Chen, "Fault diagnosis based on Littlewood-Paley wavelet support vector machine," Journal of Vibration and Shock, vol. 30, no. 1, pp. 128-131, 2011.

[11] H. Shuixia, Z. Guangming, and Q. Chunling, "The elevator fault diagnosis system based on KPCA and SVM," Machinery Design \& Manufacture, vol. 1, pp. 196-198, 2010.

[12] F. Li, B.-P. Tang, and W.-Y. Liu, "Fault diagnosis based on least square support vector machine optimized by genetic algorithm," Journal of Chongqing University (Natural Science Edition), vol. 33, no. 12, pp. 14-20, 2010.

[13] A. Widodo and B.-S. Yang, "Support vector machine in machine condition monitoring and fault diagnosis," Mechanical Systems and Signal Processing, vol. 21, no. 6, pp. 2560-2574, 2007.

[14] W. Di, A study on the multi-parameter embedded type onsite measurement system for elevator rails [M.S. thesis], Tianjin University, Tianjin, China, 2003.

[15] C. Li, The Analysis on the Dynamic Characteristics of Elevator System and the Research on the Method of Fault Diagnosis, Zhejiang University, Hangzhou, China, 1997. 
[16] C. Xiang, "The analysis on the vibration of elevator operation," China High-Tech Enterprises, vol. 21, pp. 60-62, 2012.

[17] L. Zhuocheng, "The analysis on the causes and measures of elevator car vibration," China New Technologies and Products, vol. 23, pp. 113-115, 2012.

[18] S. Dianbin, "The discussion on the reason and solution of elevator shaking," Heilongjiang Science and Technology Information, vol. 12, pp. 45-46, 2011.

[19] Y. Yunlong, "Fault diagnosis of rolling bearing based on kurtosis-wavelet packet analysis," New Technology \& New Process, vol. 5, pp. 43-46, 2008.

[20] V. Vapnik, The Nature of Statistical Learning Theory, Springer, New York, NY, USA, 2nd edition, 1998.

[21] B. Jiang, W. Liu, Z. Dai, and H. Wang, "Working condition based optimization framework for operational patterns and its application in petrochemical industry," CIESC Journal, vol. 63, no. 12, pp. 3978-3984, 2012.

[22] L. Ma, H. Zhang, H. Zhou, and Q. He, "Flow regime identification of oil-water two-phase flow based on HHT and SVM," Journal of Chemical Industry and Engineering, vol. 58, no. 3, pp. 617-622, 2007 (Chinese).

[23] S. Tao, D. Chen, and W. Hu, "Gradient algorithm for selecting hyper parameters of LSSVM in process modeling," Journal of Chemical Industry and Engineering, vol. 58, no. 6, pp. 1514-1517, 2007 (Chinese).

[24] Q.-M. Zhang and H.-J. Liu, "Application of LS-SVM in classification of power quality disturbances," Proceedings of the Chinese Society of Electrical Engineering, vol. 28, no. 1, pp. 106-110, 2008.

[25] Q. Wang and X. Tian, "Soft sensing based on KPCA and LSSVM,” CIESC Journal, vol. 62, no. 10, pp. 2813-2817, 2011. 

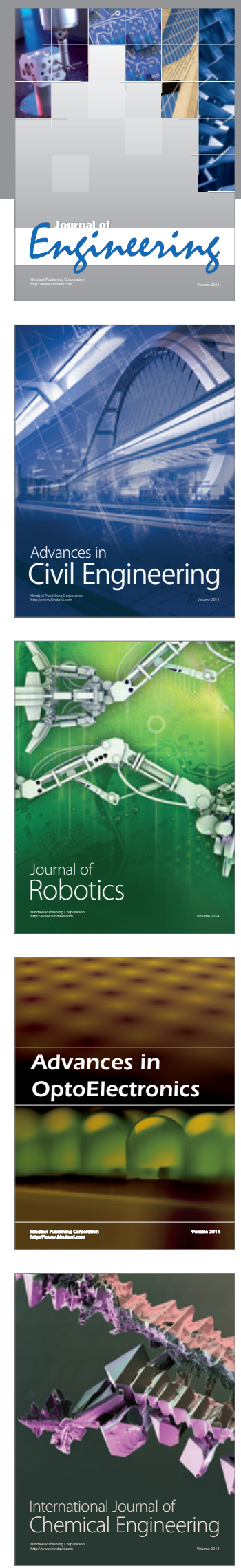

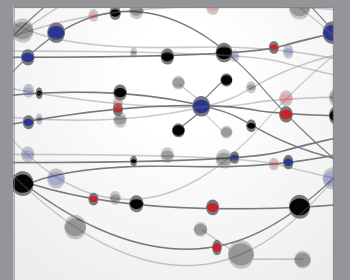

The Scientific World Journal
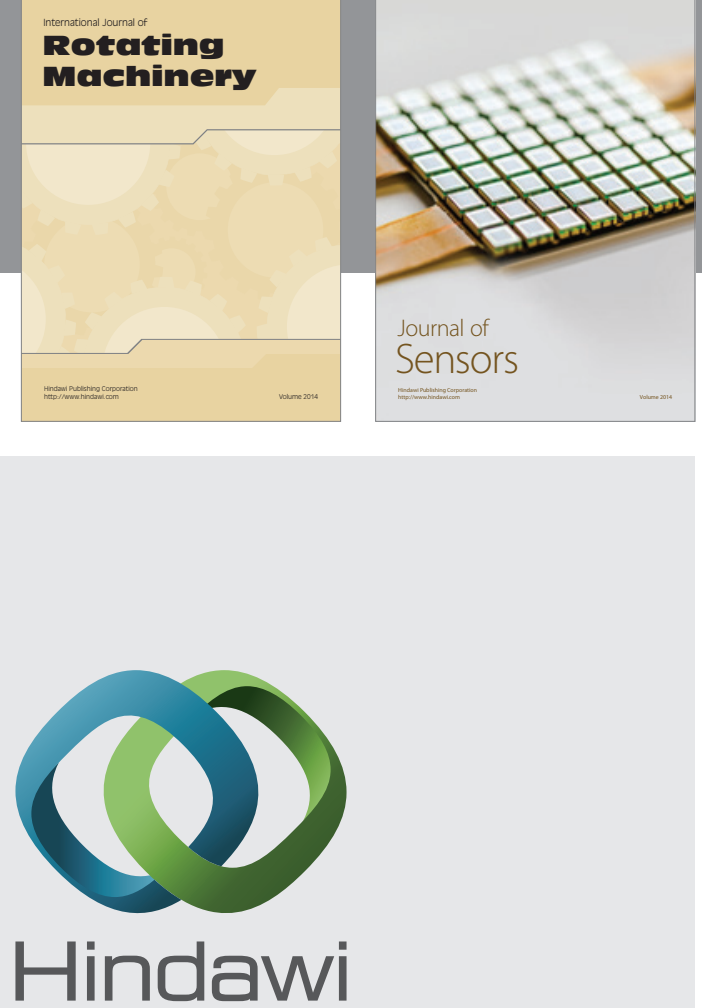

Submit your manuscripts at http://www.hindawi.com
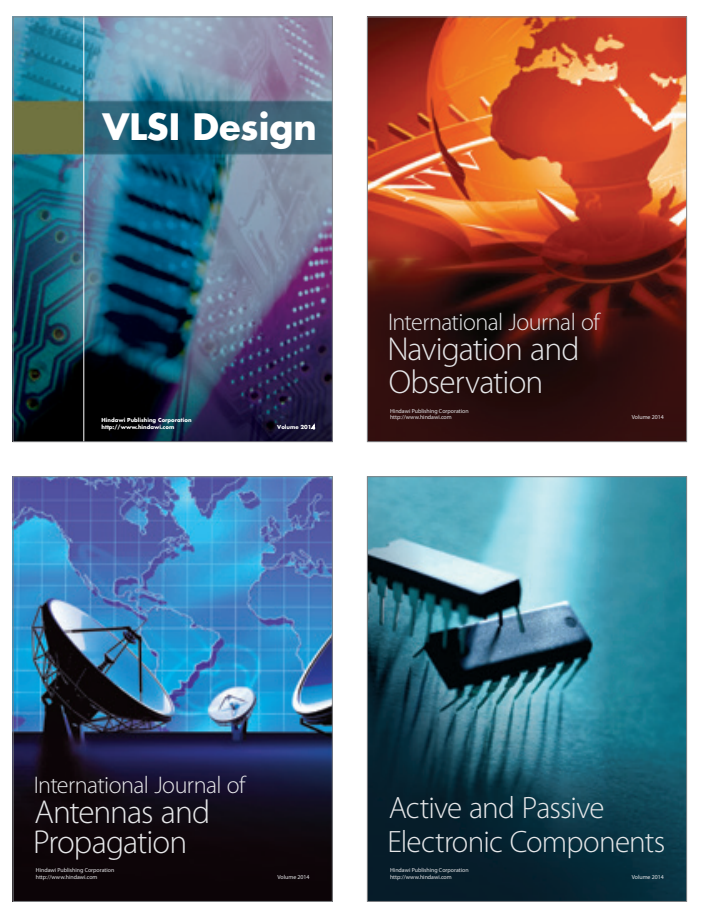
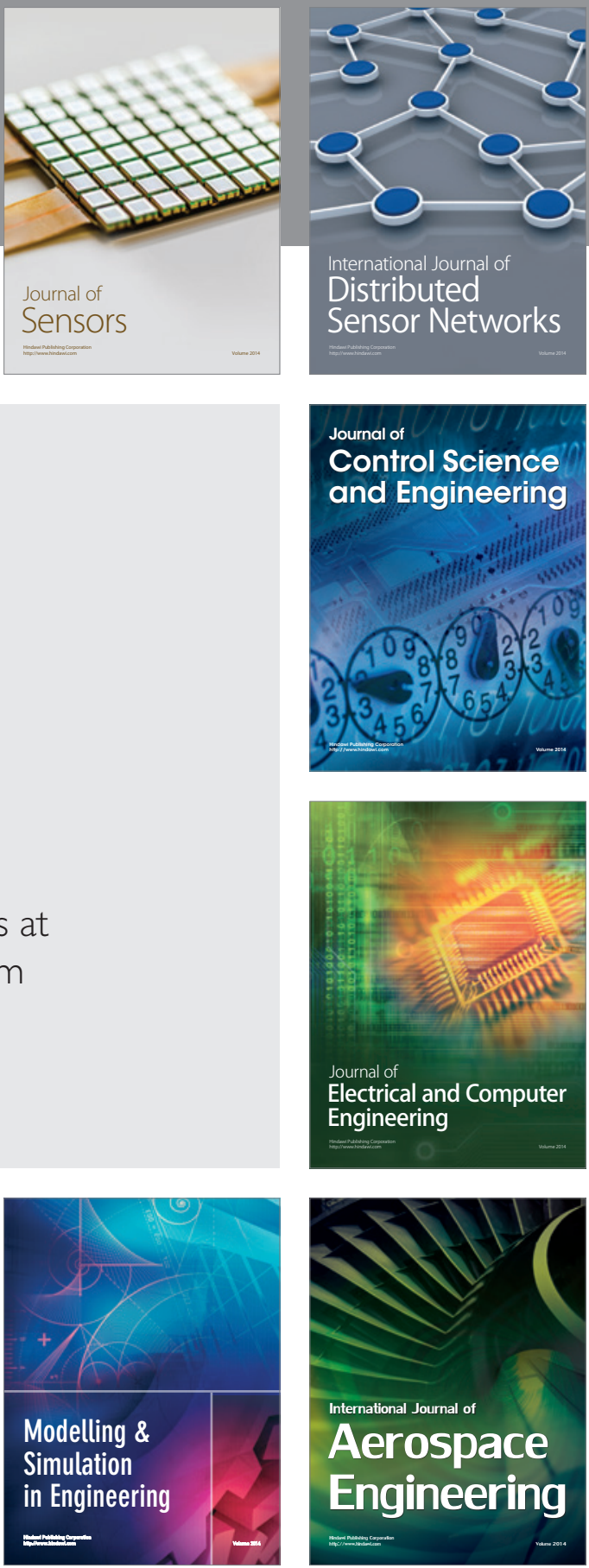

Journal of

Control Science

and Engineering
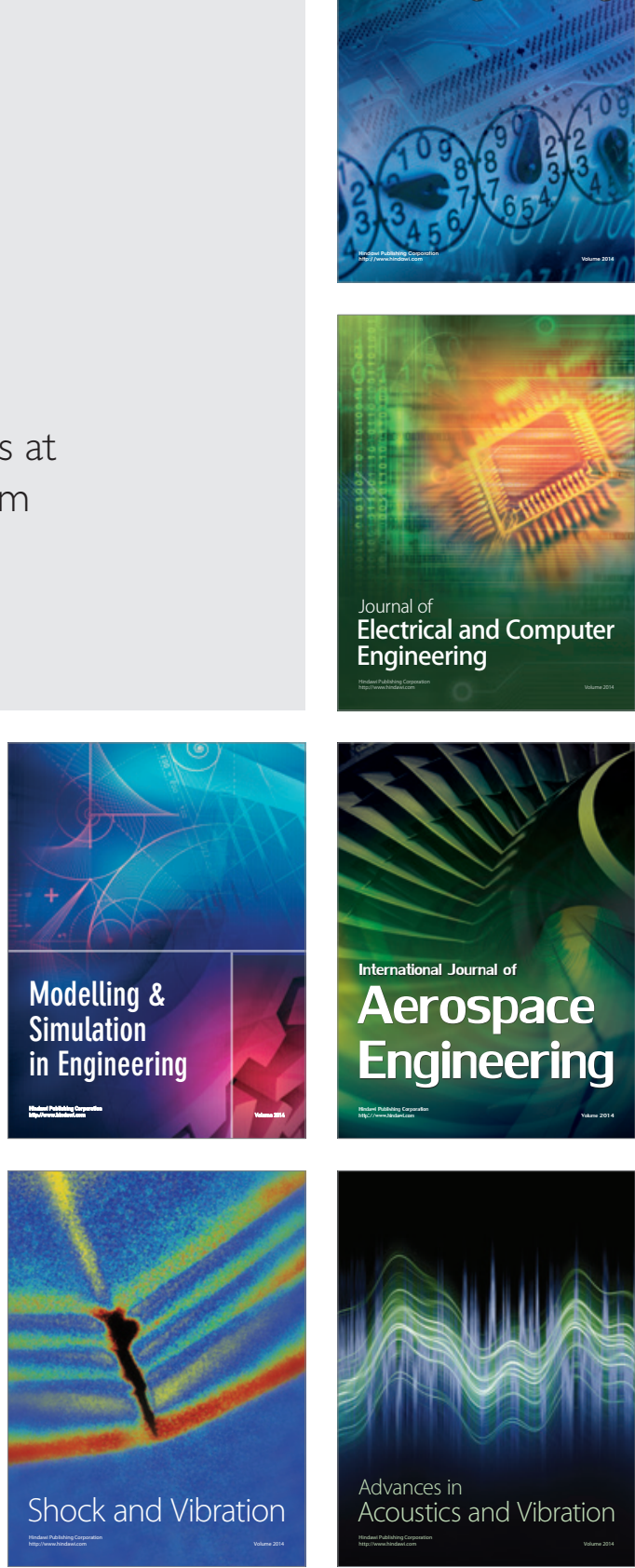